\title{
Synthesis of Some New 3-Pyrrolidinylquinoline Derivatives via 1,3-Dipolar Cycloaddition of Stabilized Azomethine Ylides to Quinolinyl $\alpha, \beta$ - Unsaturated Ketones
}

\author{
A. Bouraiou ${ }^{1}$, A. Debache ${ }^{1}$, S. Rhouati ${ }^{1}$, A. Belfaitah*,1, N. Benali-Cherif ${ }^{2}$ and B. Carboni ${ }^{3}$ \\ ${ }^{1}$ Laboratoire des Produits Naturels d'Origine Végétale et de Synthèse Organique, Faculté des Sciences Exactes, \\ Campus de Chaabat Ersas, Université Mentouri-Constantine, 25000, Algeria \\ ${ }^{2}$ Laboratoire des Structures, Propriétés et Interactions Inter Atomiques (LASPI ${ }^{2}$ A), Centre Universitaire de Khenchela, \\ 40000 Khenchela, Algéria \\ ${ }^{3}$ Sciences Chimiques de Rennes, UMR 6226 CNRS-Université de Rennes 1, Bat 10 A, Campus de Beaulieu, 35042 \\ Rennes CEDEX, France
}

\begin{abstract}
N-Metallated azomethine ylide generated from methyl $(E)-N$-benzylideneglycinate, $\mathrm{LiBr}$ and triethylamine underwent cycloaddition to quinolyl $\alpha, \beta$-unsaturated ketones with excellent diastereoselectivity to afford new functionalised 3-pyrrolidinylquinoline derivatives.
\end{abstract}

Keywords: 1,3-dipolar cycloaddition, quinoline, pyrrolidine synthesis, azomethine ylides.

\section{INTRODUCTION}

Quinolines derivatives have attracted considerable interest for many years due to their presence in the skeleton of a large number of bioactive compounds and natural products [1]. For example, quinoline alkaloids, such as quinine, chloroquine, mefloquine and amodiaquine, are used as efficient drugs for the treatment of malaria [2].

On the other hand 1,3-dipolar cycloaddition reactions of azomethine ylides with olefinic dipolarophiles had resulted in a number of novel heterocyclic scaffolds which are particularly useful for the creation of diverse chemical libraries of drug-like molecules for biological screening [3]. Functionalized pyrrolidine containing compounds are also of significant importance because of their biological activities and widespread employment in catalysis [4].

The coupling of this chemical entity with quinoline unit might as well be envisioned to bring with some biological activities. In this context, some limited investigations have been carried out which involved the combination of the quinolyl moiety and the pyrrolidine unit.

As a part of our program related to the preparation and biological evaluation of quinolyl derivatives [5], we have recently described a practical and an efficient synthesis of some 3-pyrrolidinylquinoline derivatives from quinolinyl $\alpha, \beta$-unsaturated esters as starting materials via 1,3-dipolar cycloaddition [6]. In a continuation of our efforts in this area, we report here an efficient procedure for the preparation of new pyrrolidine derivatives bearing a quinoline ring at $\mathrm{C}-3$,

*Address correspondence to this author at the Laboratoire des Produits Naturels d'Origine Végétale et de Synthèse Organique, Faculté des Sciences Exactes, Campus de Chaabat Ersas, Université Mentouri-Constantine, 25000, Algeria; Tel/Fax: 00213 (0)31 8188 62;

E-mail: abelbelfaitah@yahoo.fr aroyl or acetyl group at C-4, and a phenyl substituent at C-5 via an 1,3-dipolar cycloaddition reaction of a stabilized metallo-azomethine ylide to quinolinyl $\alpha, \beta$-unsaturated ketones [7].

\section{RESULTS AND DISCUSSION}

Starting from the corresponding 2-chloro-3formylquinoline derivatives $\mathbf{1}$, chalcones $\mathbf{2 a - 2 h}$ were synthesized by Claisen-Schmidt condensation reactions of appropriately substituted acetophenone in ethanol in the presence of $10 \%$ of aqueous $\mathrm{NaOH}$ [8]. The methylketone derivatives $\mathbf{2 i - 2 j}$ were prepared from the aldehydes $\mathbf{1}$ via a Wittig reaction using methyl(triphenylphosphoranylidene) acetate and were obtained in good yields (Scheme 1).

The E-configurated dipolarophiles $(\mathbf{2 a - 2 j})$ reacted with azomethine ylide, generated from methyl (E)-Nbenzylideneglycinate in the presence of $\mathrm{LiBr}$ and triethylamine at room temperature, employing dry THF as the solvent (Scheme 2).

In accordance with literature reports $[9,10]$, this 1,3dipolar cycloaddition reaction of the in situ generated metallo-azomethine ylide, exhibited high regio and stereoselectivity leading to the expected syn-endo cycloadduct (3a-3j).

All results reported below shown that pyrrolidines were obtained with conservation of the stereochemistry of starting alkenes [11], giving only one diastereoisomer with no evidence of any other isomers in the ${ }^{1} \mathrm{H}$ NMR spectra of the crude products (Table $\mathbf{1}$ ).

The structure of compounds $3 \mathrm{a}-3 \mathrm{j}$ has been established by analogy and by comparison of their ${ }^{1} \mathrm{H}$ NMR with those reported [12]. The shielding of the protons of the methyl connected to the aroyl group attached at $\mathrm{C}-4$ by the adjacent 5 -phenyl ring $(\delta=1.83 \mathrm{ppm})$ confirms the regiochemistry and demonstrated the 4,5-cis configuration relationship [6,13]. 
<smiles>[R]c1c([R])c([R])c2nc(Cl)c(C=O)cc2c1[R]</smiles>

1a-1e

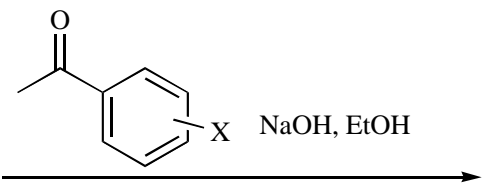

Or $\mathrm{Ph}_{3} \mathrm{PCH}=\mathrm{COR}$, DME<smiles>[R]C(=O)/C=C/c1cc2c([R])c([R])c([R])c([R])c2nc1Cl</smiles>

$\mathbf{2 a - 2 j}$

Scheme 1. Synthesis of chalcone and methyl(vinylquinoline) ketone derivatives.<smiles></smiles>

$2 \mathbf{a}-2 \mathbf{j}$

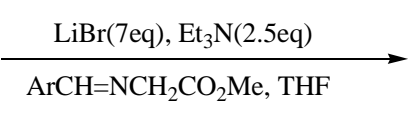

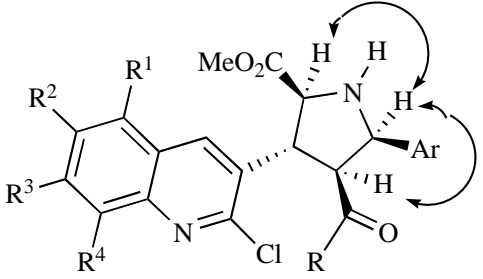

3a-3j

Scheme 2. Synthesis of quinolylpyrrolidine N-H derivatives (3a-3j).

Table 1. Synthesis of 3-pyrrolidinylquinolines 3

\begin{tabular}{|c|c|c|c|c|c|c|}
\hline Compound & $\mathbf{R}^{1}$ & $\mathbf{R}^{2}$ & $\mathbf{R}^{3}$ & $\mathbf{R}^{4}$ & $\mathbf{R}$ & Yield (\%) \\
\hline $3 \mathbf{b}$ & $\mathrm{H}$ & $\mathrm{H}$ & $\mathrm{H}$ & $\mathrm{H}$ & $2-\mathrm{MeC}_{6} \mathrm{H}_{4}$ & 58 \\
\hline $3 \mathrm{c}$ & $\mathrm{OMe}$ & $\mathrm{H}$ & $\mathrm{H}$ & $\mathrm{OMe}$ & 2- $\mathrm{MeC}_{6} \mathrm{H}_{4}$ & 71 \\
\hline 3d & $\mathrm{H}$ & $\mathrm{Me}$ & $\mathrm{H}$ & $\mathrm{H}$ & 2- $\mathrm{MeC}_{6} \mathrm{H}_{4}$ & 62 \\
\hline 3f & $\mathrm{H}$ & $\mathrm{OMe}$ & $\mathrm{H}$ & $\mathrm{H}$ & $4-\mathrm{OMeC}_{6} \mathrm{H}_{4}$ & 65 \\
\hline $3 g$ & $\mathrm{H}$ & $\mathrm{H}$ & $\mathrm{H}$ & $\mathrm{Me}$ & 3,4-diMeOC $6 \mathrm{H}_{3}$ & 53 \\
\hline $3 \mathbf{h}$ & $\mathrm{H}$ & $\mathrm{H}$ & $\mathrm{H}$ & $\mathrm{Me}$ & 3,4,5-triMeOC ${ }_{6} \mathrm{H}_{2}$ & 54 \\
\hline $3 \mathbf{i}$ & $\mathrm{H}$ & $\mathrm{Me}$ & $\mathrm{H}$ & $\mathrm{H}$ & $\mathrm{Me}$ & 67 \\
\hline
\end{tabular}

The structure of compound 3a, as representative example, was elucidated by detailed NMR studies (Table 2). The ${ }^{1} \mathrm{H}$ and ${ }^{13} \mathrm{C}$ NMR assignments were made on the basis of high-field one and two-dimensional methods (HSQC, COSY, and NOESY H, H). The '2,4,5-cis' configuration of these pyrrolidines was confirmed by the observed NOE en- hancement between the two pairs $(\mathrm{H}-2$ and $\mathrm{H}-5)$ and $(\mathrm{H}-2$ and $\mathrm{H}-4$ ) (Table 2).

X-ray crystallography of $\mathbf{3 e}$ (Fig. 1) showed an asymmetric unit which contains two independent molecules and the analysis demonstrate that the two stereoisomers have for each one, the absolute stereochemistry $(2 S, 3 R, 4 S, 5 R)$ and

Table 2. Significant ${ }^{1} \mathrm{H},{ }^{13} \mathrm{C}$ NMR Data, Selected H-H Coupling NOE for 3a

\begin{tabular}{|c|c|c|c|c|}
\hline & $\boldsymbol{\delta}^{13} \mathbf{C}$ & $\boldsymbol{\delta}^{\mathbf{1}} \mathbf{H}(\mathbf{m}, \mathbf{J})$ & ${ }^{1} \mathbf{H}\left\{{ }^{\mathbf{1}} \mathbf{H}\right\} \mathbf{n} . \mathbf{O} . \mathbf{e}^{\mathbf{a}}$ & ${ }^{\mathbf{1}} \mathbf{H},{ }^{\mathbf{1}} \mathbf{H} \mathbf{c o s y}$ \\
\hline \hline H-2 & 66.5 & $4.40(\mathrm{~d}, 7.9)$ & H-5, H-4 Qui, 2-CO 2 Me & 4.69 \\
H-5 & 66.4 & $4.96(\mathrm{~d}, 8.1)$ & H-2, H-6 Ar, H-2 Ar & 4.77 \\
H-4 & 60.8 & $4.77(\mathrm{t}, 8.0)$ & H-2, H-4 Qui & $4.69,4.96$ \\
H-3 & 50.5 & $4.69(\mathrm{t}, 8.0)$ & H-4 Qui & $4.40,4.77$ \\
\hline
\end{tabular}

${ }^{a}$ Obtained by 2D-NOESY spectroscopy. 
$(2 R, 3 S, 4 R, 5 S)$ of the new stereocenters created in the cycloaddition reactions [14].

Fig. (1). ORTEP of asymmetric unit of compound 3e which contains two independent molecules projection down (010).

\section{CONCLUSIONS}

In conclusion, we report herein an efficient approach to 3 -pyrrolidinylquinoline derivatives that exploits [3+2] cycloaddition reactions of azomethine ylides. This approach allows a diverse range of compounds to be generated in good yield and the pharmacological actions of the new pyrrolidine derivatives will be inspected afterwards.

\section{EXPERIMENTAL SECTION}

\section{General Information}

THF was freshly distilled from sodium/benzophenone, $\mathrm{POCl}_{3}$ and $\mathrm{CH}_{2} \mathrm{Cl}_{2}$ from $\mathrm{P}_{2} \mathrm{O}_{5}$, DMF was kept for few hours over $\mathrm{CaCl}_{2}$ and distilled from $\mathrm{CaO}$ and DME from $\mathrm{NaH}$. $\mathrm{EtOH}$ was distilled from magnesium. Melting points were determined on an Electrothermal Digital Melting Points Apparatus IA 9200 and are uncorrected. I.R spectra were performed on Shimadzu FT IR-8201 PC spectrophotometer and Perkin Elmer Spectrum One (FT-IR) spectrophotometer with a universal ATR sampling accessory. NMR spectra were recorded in $\mathrm{CDCl}_{3}$ on a Brüker Avance DPX250 or Brüker Avance DMX300 spectrometer. Chemical shifts $(\delta)$ are given in ppm and $\mathbf{J}$ values in Hertz $(\mathrm{Hz})$. column chromatography was performed on Merck silica gel (60, particle size 0.063-0.2 mm) using $\mathrm{CHCl}_{3}$ or $\mathrm{CH}_{2} \mathrm{Cl}_{2}$ as eluent. Thin layer chromatography (TLC) was carried out on precoated Merck silica gel aluminium sheets $60 \mathrm{~F}_{254}$. HRMS data were obtained on spectrometer MAT 311 (Centre Régional de Mesures Physiques de l'Ouest). X-Ray crystallographic analysis was performed with an Enraf-Nonius KAPPA CCD at 293 K using Mo $\mathrm{K} \alpha$ radiation $(\lambda=0.71073 \AA)$.

Substituted 2-chloroquinolyl-3-carbaldehydes have been synthesized according to reported methods [15]. Methyl benzylidene aminoacetate is obtained by treatment of benzaldehyde with methyl glycinate hydrochloride in basic medium [16].
General Method for the Synthesis of Chalcone Derivatives (2a-2h)

To a solution of $10 \% \mathrm{NaOH}(520 \mathrm{mg}, 13 \mathrm{mmol})$ in $95 \%$ ethanol $(20 \mathrm{~mL})$ was added $500 \mathrm{mg}(2.61 \mathrm{mmol})$ of 2-chloro3 -formylquinoline and the acetophenone derivative (1.0 eq., $2.61 \mathrm{mmol})$. The mixture was stirred at $25{ }^{\circ} \mathrm{C}$ for $24 \mathrm{~h}$. The contents were then cooled and poured into cold water then neutralized with dilute $\mathrm{HCl}$. The solid obtained was filtered, washed, and dried on air to afford the crude chalcone.

(E)-3-(2-Chloro-8-methylquinolin-3-yl)-1-o-tolylprop-2-en1-one $(2 a)$

Yd 94\%. $\mathrm{R}_{f}\left(\mathrm{CH}_{2} \mathrm{Cl}_{2}\right): 0.60 . \mathrm{Mp} 165-167{ }^{\circ} \mathrm{C}$. IR $v_{\max }$ (KBr) $1645 \mathrm{~cm}^{-1}\left(\mathrm{C}=\mathrm{O}\right.$, ketone). ${ }^{1} \mathrm{H}$ NMR $(250 \mathrm{MHz}$, $\left.\mathrm{CDCl}_{3}\right) \delta 8.48(\mathrm{~s}, 1 \mathrm{H}), 8.00(\mathrm{~d}, J=16.1,1 \mathrm{H}), 7.75(\mathrm{~d}, J=8.9$, $1 \mathrm{H}), 7.52(\mathrm{dd}, J=9.1, J=2.4,1 \mathrm{H}), 7.50-7.43(\mathrm{~m}, 3 \mathrm{H}), 7.40$ (ddd, $J=8.9, J=8.1, J=2.3,1 \mathrm{H}), 7.38(\mathrm{dd}, J=9.4, J=1.2,1 \mathrm{H})$, $7.25(\mathrm{~d}, J=16.1,1 \mathrm{H}), 2.75(\mathrm{~s}, 3 \mathrm{H}), 2.50(\mathrm{~s}, 3 \mathrm{H}) .{ }^{13} \mathrm{C} \mathrm{NMR}$ $\left(62.5 \mathrm{MHz}, \mathrm{CDCl}_{3}\right) \delta 195.2(\mathrm{C}=\mathrm{O}), 149.1(\mathrm{C}), 147.1(\mathrm{C})$, $140.5(\mathrm{CH}), 138.2(\mathrm{C}), 137.4(\mathrm{C}), 136.7(\mathrm{CH}), 136.4(\mathrm{CH})$, $131.7(\mathrm{C}), 131.5(\mathrm{CH}), 130.9(\mathrm{CH}), 130.2(\mathrm{C}), 128.4(\mathrm{CH})$, $127.4(\mathrm{CH}), 127.3(\mathrm{C}), 127.0(\mathrm{CH}), 125.8(\mathrm{CH}), 125.5(\mathrm{CH})$, $20.4\left(\mathrm{CH}_{3}\right), 17.7\left(\mathrm{CH}_{3}\right)$. HRMS (EI): $\mathrm{m} / \mathrm{z}\left[\mathrm{M}^{+}\right]$Calcd. for $\mathrm{C}_{20} \mathrm{H}_{16} \mathrm{NO}^{35} \mathrm{Cl}$ : 321.09204, found 321.0928.

(E)-3-(2-Chloroquinolin-3-yl)-1-o-tolylprop-2-en-1-one (2b)

Yd 88\%. $\quad \mathrm{R}_{f}\left(\mathrm{CH}_{2} \mathrm{Cl}_{2}\right): 0.65 . \quad \mathrm{Mp} \mathrm{120-124}{ }^{\circ} \mathrm{C}$. IR $v_{\max }(\mathrm{KBr}) 1685 \mathrm{~cm}^{-1}\left(\mathrm{C}=\mathrm{O}\right.$, ketone). ${ }^{1} \mathrm{H}$ NMR $(250 \mathrm{MHz}$, $\left.\mathrm{CDCl}_{3}\right) \delta 8.50(\mathrm{~s}, 1 \mathrm{H}), 7.95(\mathrm{~d}, J=15.9,1 \mathrm{H}), 7.79$ (dd, $J=8.7$, $J=8.4,1 \mathrm{H}), 7.52(\mathrm{dd}, J=8.9, J=2.4,1 \mathrm{H}), 7.50-7.32(4 \mathrm{H}, \mathrm{m})$, $7.37(\mathrm{dd}, J=8.4, J=1.2,1 \mathrm{H}), 7.29(\mathrm{~d}, J=15.9,1 \mathrm{H}), 6.82(\mathrm{td}$, $J=9.1, J=2.1,1 \mathrm{H}), 2.50(\mathrm{~s}, 3 \mathrm{H}) .{ }^{13} \mathrm{C} \mathrm{NMR}(62.5 \mathrm{MHz}$, $\left.\mathrm{CDCl}_{3}\right) \delta 195.2(\mathrm{C}=\mathrm{O}), 150.2(\mathrm{C}), 147.8(\mathrm{C}), 140.1(\mathrm{CH})$, $138.1(\mathrm{C}), 137.5(\mathrm{C}), 137.3(\mathrm{CH}), 136.1(\mathrm{CH}), 133.4(\mathrm{CH})$, $132.0(\mathrm{CH}), 131.6(\mathrm{CH}), 131.4(\mathrm{CH}), 131.3(\mathrm{C}), 130.6(\mathrm{CH})$, $129.9(\mathrm{CH}), 127.2(\mathrm{C}), 125.5(\mathrm{CH}), 124.8(\mathrm{CH}), 20.4\left(\mathrm{CH}_{3}\right)$. HRMS (EI): m/z [ $\left.\mathrm{M}^{+}\right]$Calcd. for $\mathrm{C}_{19} \mathrm{H}_{14} \mathrm{NO}^{35} \mathrm{Cl}$ : 307.07639, found 307.0769 .

(E)-3-(2-Chloro-5,8-dimethoxyquinolin-3-yl)-1-o-tolylprop2-en-1-one (2c)

Yd 87\%. $\quad \mathrm{R}_{f}\left(\mathrm{CH}_{2} \mathrm{Cl}_{2}\right):$ 0.62. Mp 169-170 ${ }^{\circ} \mathrm{C} . \quad \mathrm{IR}$ $v_{\max }(\mathrm{KBr}) 1672 \mathrm{~cm}^{-1}\left(\mathrm{C}=\mathrm{O}\right.$, ketone). ${ }^{1} \mathrm{H}$ NMR $(300 \mathrm{MHz}$, $\left.\mathrm{CDCl}_{3}\right) \delta 8.90(\mathrm{~s}, 1 \mathrm{H}), 7.97(\mathrm{~d}, J=16.0,1 \mathrm{H}), 7.60(\mathrm{~d}, J=7.5$, $1 \mathrm{H}), 7.49$ (d, $J=7.4,1 \mathrm{H}), 7.19-7.42(\mathrm{~m}, 3 \mathrm{H}), 7.05$ (d, $J=16.0$, $1 \mathrm{H}), 6.89(\mathrm{~d}, J=8.6,1 \mathrm{H}), 4.04(\mathrm{~s}, 3 \mathrm{H}), 4.00(\mathrm{~s}, 3 \mathrm{H}), 2.51(\mathrm{~s}$, $3 \mathrm{H}) .{ }^{13} \mathrm{C}$ NMR $\left(75.4 \mathrm{MHz}, \mathrm{CDCl}_{3}\right) \delta 197.5(\mathrm{C}=\mathrm{O}), 151.3(\mathrm{C})$, $148.9(\mathrm{C}), 148.4(\mathrm{C}), 140.6(\mathrm{CH}), 138.7(\mathrm{C}), 135.6(\mathrm{CH})$, $132.1(\mathrm{C}), 131.5(\mathrm{C}), 131.5(\mathrm{CH}), 130.9(\mathrm{CH}), 130.4(\mathrm{C})$, $128.4(\mathrm{CH}), 127.9(\mathrm{CH}), 125.5(\mathrm{CH}), 121.8(\mathrm{C}), 109.8(\mathrm{CH})$, $104.8(\mathrm{CH}), 56.2\left(\mathrm{OCH}_{3}\right), 55.8\left(\mathrm{OCH}_{3}\right), 20.4\left(\mathrm{CH}_{3}\right)$. HRMS (EI): $\mathrm{m} / \mathrm{z}\left[\mathrm{M}^{+}\right]$Calcd. for $\mathrm{C}_{21} \mathrm{H}_{18} \mathrm{NO}_{3}{ }^{35} \mathrm{Cl}$ : 367.09752, found 367.0961 .

(E)-3-(2-Chloro-6-methylquinolin-3-yl)-1-o-tolylprop-2-en1-one (2d)

Yield 91\%. $\mathrm{R}_{f}\left(\mathrm{CH}_{2} \mathrm{Cl}_{2}\right):$ 0.59. Mp 143-145 ${ }^{\circ} \mathrm{C}$. IR $v_{\max }(\mathrm{KBr}) 1654 \mathrm{~cm}^{-1}\left(\mathrm{C}=\mathrm{O}\right.$, ketone). ${ }^{1} \mathrm{H}$ NMR $(250 \mathrm{MHz}$, $\left.\mathrm{CDCl}_{3}\right) \delta 8,41(\mathrm{~s}, 1 \mathrm{H}), 7.90(\mathrm{~d}, J=16.0,1 \mathrm{H}), 7.85(\mathrm{~d}, J=8.3$, $1 \mathrm{H}), 7.60-7.55(\mathrm{~m}, 5 \mathrm{H}), 7.32(\mathrm{~d}, J=16.0,1 \mathrm{H}), 7.00(\mathrm{dd}$, $J=8.4, J=2.2,1 \mathrm{H}), 2.43(\mathrm{~s}, 3 \mathrm{H}), 2.66(\mathrm{~s}, 3 \mathrm{H}) .{ }^{13} \mathrm{C}$ NMR $(62.9$ $\left.\mathrm{MHz} \mathrm{CDCl}_{3}\right) \delta 197.8(\mathrm{C}=\mathrm{O}), 150.1(\mathrm{C}), 146.6(\mathrm{C}), 140.5$ 
$(\mathrm{CH}), 138.2(\mathrm{C}), 136.0(\mathrm{C}), 136.5(\mathrm{CH}), 135.4(\mathrm{CH}), 131.7$ (C), $131.5(\mathrm{CH}), 130.9(\mathrm{CH}), 130.2(\mathrm{C}), 128.4(\mathrm{CH}), 128.1$ $(\mathrm{CH}), 127.4(\mathrm{C}), 127.0(\mathrm{CH}), 125.6(\mathrm{CH}), 125.2(\mathrm{CH}), 20.4$ $\left(\mathrm{CH}_{3}\right), 18.1\left(\mathrm{CH}_{3}\right)$. HRMS (EI): $\mathrm{m} / \mathrm{z}\left[\mathrm{M}^{+}\right]$Calcd. for $\mathrm{C}_{20} \mathrm{H}_{16} \mathrm{NO}^{35} \mathrm{Cl}: 321.09204$, found 321.0928 .

(E)-3-(2-Chloro-8-methylquinolin-3-yl)-1-(4-methoxyphenyl) prop-2-en-1-one (2e)

Yield 97\%. $\mathrm{R}_{f}\left(\mathrm{CH}_{2} \mathrm{Cl}_{2}\right): 0.55 . \mathrm{Mp} \mathrm{131-134}{ }^{\circ} \mathrm{C}$. IR $v_{\max }(\mathrm{KBr}) 1662 \mathrm{~cm}^{-1}\left(\mathrm{C}=\mathrm{O}\right.$, ketone). ${ }^{1} \mathrm{H}$ NMR $(250 \mathrm{MHz}$, $\left.\mathrm{CDCl}_{3}\right) \delta 8.50(\mathrm{~s}, 1 \mathrm{H}), 8.15(\mathrm{~d}, J=15.7,1 \mathrm{H}), 8.14(\mathrm{~d}, J=8.8$, $2 \mathrm{H})$, 7.77-7.67 (m, 3H), $7.60(\mathrm{~d}, J=15.7,1 \mathrm{H}), 7.00(\mathrm{~d}, J=8.8$, 2H), $3.96(\mathrm{~s}, 3 \mathrm{H}), 2.75(\mathrm{~s}, 3 \mathrm{H}) .{ }^{13} \mathrm{C} \mathrm{NMR}(62.9 \mathrm{MHz}$, $\left.\mathrm{CDCl}_{3}\right) \delta 187.7(\mathrm{C}=\mathrm{O}), 163.6(\mathrm{C}), 149.7(\mathrm{C}), 149.3(\mathrm{C})$, $147.0(\mathrm{C}), 138.7(\mathrm{CH}), 136.6(\mathrm{CH}), 136.3(\mathrm{C}), 131.5(\mathrm{CH})$, $131.0(\mathrm{CH}), 130.5(\mathrm{C}), 130.3(\mathrm{CH}), 127.7(2 \times \mathrm{CH}), 127.3$ $(\mathrm{CH}), 127.0(\mathrm{C}), 113.9(2 \times \mathrm{CH}), 55.5\left(\mathrm{OCH}_{3}\right), 17.7\left(\mathrm{CH}_{3}\right)$. HRMS (EI): $\mathrm{m} / \mathrm{z}\left[\mathrm{M}^{+}\right]$Calcd. for $\mathrm{C}_{20} \mathrm{H}_{16} \mathrm{NO}_{2}{ }^{35} \mathrm{Cl}$ : 337.08696 , found 337.0869 .

(E)-3-(2-Chloro-6-methoxyquinolin-3-yl)-1-(4-methoxyphenyl)prop-2-en-1-one (2f)

Yield 90\%. $\mathrm{R}_{f}\left(\mathrm{CH}_{2} \mathrm{Cl}_{2}\right)$ : 0.65. Mp 142-145 ${ }^{\circ} \mathrm{C}$. IR $v_{\max }(\mathrm{KBr}) 1653 \mathrm{~cm}^{-1}\left(\mathrm{C}=\mathrm{O}\right.$, ketone). ${ }^{1} \mathrm{H}$ NMR $(250 \mathrm{MHz}$, $\left.\mathrm{CDCl}_{3}\right) \delta 8.56(\mathrm{~s}, 1 \mathrm{H}), 8.21(\mathrm{~d}, J=15.9,1 \mathrm{H}), 7.57(\mathrm{~d}, J=8.1$, $1 \mathrm{H}), 7.55(\mathrm{~d}, J=8.7,2 \mathrm{H}), 7.22(\mathrm{~d}, J=8.9,1 \mathrm{H}), 7.15(\mathrm{~d}$, $J=15.9,1 \mathrm{H}), 7.12(\mathrm{~s}, 1 \mathrm{H}) 6.75(\mathrm{~d}, J=8.6,2 \mathrm{H}), 4.00(\mathrm{~s}, 3 \mathrm{H})$, $3.73(\mathrm{~s}, 3 \mathrm{H}) .{ }^{13} \mathrm{C}$ NMR $\left(75.4 \mathrm{MHz}, \mathrm{CDCl}_{3}\right) \delta 195.8(\mathrm{C}=\mathrm{O})$, 162.5 (C), 155.4 (C), 142.7 (C), 142.5 (C), 137.9 (C), 134.4 $(\mathrm{CH}), 132.6(\mathrm{C}), 131.5(\mathrm{CH}), 131.8(\mathrm{CH}), 127.0(\mathrm{CH}), 126.2$ (C), $125.9(2 \times C H), 121.6(\mathrm{CH}), 113.1(2 \times C H), 108.6(\mathrm{CH})$, $55.5\left(\mathrm{OCH}_{3}\right), 54.4\left(\mathrm{OCH}_{3}\right)$. HRMS (EI): m/z [M $\mathrm{M}^{+}$Calcd. for $\mathrm{C}_{20} \mathrm{H}_{16} \mathrm{NO}_{3}{ }^{35} \mathrm{Cl}$ : 353.08187, found 353.0825.

(E)-3-(2-Chloro-8-methylquinolin-3-yl)-1-(3,4-dimethoxyphenyl)prop-2-en-1-one (2g)

Yield 90\%. $\mathrm{R}_{f}\left(\mathrm{CH}_{2} \mathrm{Cl}_{2}\right)$ : 0.64. Mp 183-184 ${ }^{\circ} \mathrm{C}$. IR $v_{\max }$ (KBr) $1658 \mathrm{~cm}^{-1}\left(\mathrm{C}=\mathrm{O}\right.$, ketone). ${ }^{1} \mathrm{H}$ NMR $(250 \mathrm{MHz}$, $\left.\mathrm{CDCl}_{3}\right) \delta 8.50(\mathrm{~s}, 1 \mathrm{H}), 8.25(\mathrm{~d}, J=15.7,1 \mathrm{H}), 7.75-7.67(\mathrm{~m}$, $4 \mathrm{H}), 7.52(\mathrm{~d}, J=15.7,1 \mathrm{H}), 7.50(\mathrm{~s}, 1 \mathrm{H}), 7.00(\mathrm{~d}, J=8.4,1 \mathrm{H})$, $4.00(\mathrm{~s}, 3 \mathrm{H}), 3.96(\mathrm{~s}, 3 \mathrm{H}), 2.78(\mathrm{~s}, 3 \mathrm{H}) .{ }^{13} \mathrm{C} \mathrm{NMR}(62.9 \mathrm{MHz}$, $\left.\mathrm{CDCl}_{3}\right): \delta 195.5(\mathrm{C}=\mathrm{O}), 153.5(\mathrm{C}), 150.0(\mathrm{C}), 149.2(\mathrm{C})$, $146.9(\mathrm{C}), 145.7(\mathrm{CH}), 138.7(\mathrm{CH}), 136.6(\mathrm{C}), 136.4(\mathrm{CH})$, $136.3(\mathrm{CH}), 131.5(\mathrm{C}), 130.8(\mathrm{CH}), 128.6(\mathrm{C}), 127.7(\mathrm{CH})$, $127.3(\mathrm{C}), 124.9(\mathrm{CH}), 110.7(\mathrm{CH}), 109.8(\mathrm{CH}), 56.2$ $\left(\mathrm{OCH}_{3}\right), 56.1\left(\mathrm{OCH}_{3}\right), 17.7\left(\mathrm{CH}_{3}\right) . \mathrm{HRMS}(\mathrm{EI}): \mathrm{m} / \mathrm{z}\left[\mathrm{M}^{+}\right]$ Calcd. for $\mathrm{C}_{21} \mathrm{H}_{18} \mathrm{NO}_{3}{ }^{35} \mathrm{Cl}$ : 367.09752, found 367.0997.

(E)-3-(2-Chloro-8-methylquinolin-3-yl)-1-(3,4,5-trimethoxyphenyl)prop-2-en-1-one (2h)

Yield $72 \%$. $\mathrm{R}_{f}\left(\mathrm{CH}_{2} \mathrm{Cl}_{2}\right)$ : 0.65. Mp 122-124 ${ }^{\circ} \mathrm{C}$. IR $v_{\max }(\mathrm{KBr}) 1656 \mathrm{~cm}^{-1}$ (C=O, ketone). ${ }^{1} \mathrm{H}$ NMR $(250 \mathrm{MHz}$, $\left.\mathrm{CDCl}_{3}\right) \delta 8.49(\mathrm{~s}, 1 \mathrm{H}), 8.20(\mathrm{~d}, J=15.5,1 \mathrm{H}), 7.89(\mathrm{~d}, J=7.1$, $1 \mathrm{H}), 7.40(\mathrm{~d}, J=7.4,1 \mathrm{H}), 7.34(\mathrm{~d}, J=7.1,1 \mathrm{H}), 7.09(\mathrm{~d}$, $J=15.5,1 \mathrm{H}), 6.89$ (s, 2H), 3.97 (s, 3H), 3.93 (s, 3H), 3.83 (s, $6 \mathrm{H}), 2.65(\mathrm{~s}, 3 \mathrm{H}) ;{ }^{13} \mathrm{C} \mathrm{NMR}\left(62.9 \mathrm{MHz}, \mathrm{CDCl}_{3}\right) \delta 195.0$ (C=O), 151.7 (C), 148.9 (C), 144.3 (C), 143.5 (C) , 136.3 (C), $136.2(\mathrm{CH}), 136.0(\mathrm{C}), 132.4(\mathrm{C}), 131.3(\mathrm{CH}), 130.4$ (C), $128.9(\mathrm{CH}), 128.1(\mathrm{CH}), 127.4(\mathrm{CH}), 127.3(\mathrm{C}), 125.4$ $(\mathrm{CH}), 105.6(2 \times \mathrm{CH}), 59.9\left(\mathrm{OCH}_{3}\right), 55.9\left(\mathrm{OCH}_{3}\right), 54.9$ $\left(\mathrm{OCH}_{3}\right), 17.8\left(\mathrm{CH}_{3}\right)$. HRMS (EI): $\mathrm{m} / \mathrm{z}\left[\mathrm{M}^{+}\right]$Calcd. for $\mathrm{C}_{22} \mathrm{H}_{20} \mathrm{NO}_{4}{ }^{35} \mathrm{Cl}$ : 367.09752, found 367.0997.
General Procedure for the Preparation of Methyl(vinylquinoline) Ketone Derivatives

A suspension of the ylide $\mathrm{Ph}_{3} \mathrm{P}=\mathrm{CHCOMe}(313 \mathrm{mg}, 1.1$ $\mathrm{mmol})$ and the 2-chloro-3-formylquinoline (191.5 mg, 1.0 $\mathrm{mmol})$ in DME $(10 \mathrm{~mL})$ was refluxing for three hours. After cooling to room temperature, the mixture was filtered and the filtrate was condensed under reduced pressure. The residue was then purified by column chromatography over silica gel $\left(\mathrm{CH}_{2} \mathrm{Cl}_{2}\right)$ to give the olefinic product.

\section{(E)-4-(2-Chloro-6-methylquinolin-3-yl)but-3-en-2-one (2i)}

Yield 56\%. $\quad \mathrm{R}_{f}\left(\mathrm{CH}_{2} \mathrm{Cl}_{2}\right):$ 0.75. Mp 73-74 ${ }^{\circ} \mathrm{C}$. IR $v_{\max }(\mathrm{KBr}) 1645 \mathrm{~cm}^{-1}\left(\mathrm{C}=\mathrm{O}\right.$, ketone). ${ }^{1} \mathrm{H}$ NMR $(250 \mathrm{MHz}$, $\left.\mathrm{CDCl}_{3}\right) \delta 8.25(\mathrm{~s}, 1 \mathrm{H}), 7.90(\mathrm{~d}, J=16.3,1 \mathrm{H}), 7.73(\mathrm{~s}, 1 \mathrm{H})$, 7.57-7.54 (m, 2H), $6.75(\mathrm{~d}, J=16.3,1 \mathrm{H}), 2.52(\mathrm{~s}, 3 \mathrm{H}), 2.45$ (s, 3H); ${ }^{13} \mathrm{C}$ NMR (62.9 MHz, $\left.\mathrm{CDCl}_{3}\right) \delta 197.8(\mathrm{C}=\mathrm{O}), 149.1$ (C), $146.5(\mathrm{C}), 138.1(\mathrm{CH}), 137.9(\mathrm{C}), 135.4(\mathrm{CH}), 134.0$ $(\mathrm{CH}), 130.8(\mathrm{CH}), 128.0(\mathrm{CH}), 127.1(\mathrm{C}), 127.0(\mathrm{C}), 126.8$ $(\mathrm{CH}), 27.3\left(\mathrm{CH}_{3}\right), 21.6\left(\mathrm{CH}_{3}\right)$. HRMS (EI): m/z [M $\left.\mathrm{M}^{+}\right]$Calcd. for $\mathrm{C}_{14} \mathrm{H}_{12} \mathrm{NO}^{35} \mathrm{Cl}$ : 245.06074, found 245.0607 .

\section{(E)-4-(2-Chloroquinolin-3-yl)but-3-en-2-one (2j)}

Yield 70\%. $\mathrm{R}_{f}\left(\mathrm{CH}_{2} \mathrm{Cl}_{2}\right): 0.72 . \quad \mathrm{Mp} 175-177{ }^{\circ} \mathrm{C}$. IR $v_{\max }(\mathrm{KBr}) 1651 \mathrm{~cm}^{-1}\left(\mathrm{C}=\mathrm{O}\right.$, ketone). ${ }^{1} \mathrm{H}$ NMR $(300 \mathrm{MHz}$, $\left.\mathrm{CDCl}_{3}\right) \delta 8.42(\mathrm{~s}, 1 \mathrm{H}), 8.04(\mathrm{~d}, 8.4,1 \mathrm{H}), 7.98(\mathrm{~d}, J=16.3$, $1 \mathrm{H}), 7.88(\mathrm{~d}, J=8.2,1 \mathrm{H}), 7.80(\mathrm{t}, J=7.9,1 \mathrm{H}), 7.62(\mathrm{t}, J=7.3$, $1 \mathrm{H}), 6.82(\mathrm{~d}, J=16.3,1 \mathrm{H}), 2.48(\mathrm{~s}, 3 \mathrm{H}) ;{ }^{13} \mathrm{C}$ NMR $(75.4$ $\left.\mathrm{MHz}, \mathrm{CDCl}_{3}\right) \delta 197.6(\mathrm{C}), 148.5(\mathrm{C}), 138.0(\mathrm{CH}), 136.1$ $(\mathrm{CH}), 133.4(\mathrm{C}), 131.9(\mathrm{C}), 131.7(\mathrm{CH}), 131.0(\mathrm{C}), 128.4$ $(\mathrm{CH}), 128.0(\mathrm{CH}), 127.7(\mathrm{C}), 126.4(\mathrm{CH}), 30.9\left(\mathrm{CH}_{3}\right)$. HRMS (EI): $\mathrm{m} / \mathrm{z}\left[\mathrm{M}^{+}\right]$Calcd. for $\mathrm{C}_{13} \mathrm{H}_{10} \mathrm{NO}^{35} \mathrm{Cl}$ : 231.04509, found 231.0452 .

\section{General Procedure for the Preparation of Quinolylpyr- rolidine $\mathrm{N}-\mathrm{H}$ Derivatives}

To 1.5 eq. of lithium bromide dissolved in dry THF (e.g $0.5 \mathrm{~g}$ in $40 \mathrm{~mL}$ ) was added, under magnetic stirring and at room temperature, 1 eq. of benzylidene glycine imine, 1 eq. of substituted quinolyl $\alpha, \beta$-unsaturated ketone derivative and 1.2 eq. of dry $\mathrm{Et}_{3} \mathrm{~N}$. The reaction mixture was kept under stirring, at room temperature and the progress of the reaction was monitored by TLC still disappearance of starting product. The mixture was diluted with ether $(15 \mathrm{~mL})$ and work up by treatment with saturated aqueous ammonium chloride (10 $\mathrm{mL}$ ). The organic layers were separated and dried over anhydrous $\mathrm{MgSO}_{4}$. The filtrate was concentrated under reduced pressure and the residue was subjected to column chromatography on silica gel using $\mathrm{CHCl}_{3}$ as eluent to afford pure product.

Methyl 4-(2-methylbenzoyl)-3-(2-chloro-8-methylquinolin3-yl)-5-phenylpyrrolidine-2-carboxylate (3a)

Yield 70\%. $\mathrm{R}_{f}\left(\mathrm{CHCl}_{3}\right): 0.34 . \mathrm{Mp} \mathrm{74-76}{ }^{\circ} \mathrm{C}$. IR (ATR) v 3332, 2953, 1737, 1678, 1574, 1479, 1455, 1372, 1335, 1239, $753 \mathrm{~cm}^{-1} .{ }^{1} \mathrm{H}$ NMR $\left(300 \mathrm{MHz}, \mathrm{CDCl}_{3}\right) \delta 8.26(\mathrm{~s}, 1 \mathrm{H})$, $7.69(\mathrm{~d}, J=8.1,1 \mathrm{H}), 7.57(\mathrm{~d}, J=6.9,1 \mathrm{H}), 7.46(\mathrm{~m}, 2 \mathrm{H}), 7.27$ (td, $J=7.5, J=1.3,1 \mathrm{H}), 7.16-7.02(\mathrm{~m}, 6 \mathrm{H}), 6.99(\mathrm{~d}, \mathrm{~J}=7.5$, $1 \mathrm{H}), 4.96(\mathrm{~d}, J=8.1,1 \mathrm{H}), 4.77(\mathrm{t}, J=8.0,1 \mathrm{H}), 4.69(\mathrm{t}, J=8.0$, $1 \mathrm{H}), 4.40(\mathrm{~d}, J=7.9,1 \mathrm{H}), 3.80(\mathrm{~s}, 3 \mathrm{H}), 3.00$ (brs, $1 \mathrm{H}), 2.80(\mathrm{~s}$, $3 \mathrm{H}), 1.83(\mathrm{~s}, 3 \mathrm{H}) .{ }^{13} \mathrm{C}$ NMR $\left(75.4 \mathrm{MHz}, \mathrm{CDCl}_{3}\right) \delta 202.0$ $(\mathrm{C}=\mathrm{O}), 173.3(\mathrm{C}=\mathrm{O}), 150.0(\mathrm{C}), 146.3(\mathrm{C}), 139.8(\mathrm{C}), 139.0$ (C), 137.9 (C), 137.8 (CH), 136.9 (C), 132.4 (C), 132.2 
$(\mathrm{CH}), 131.8(\mathrm{CH}), 130.9(\mathrm{CH}), 129.0(\mathrm{CH}), 128.7(2 \times \mathrm{CH})$, $128.1(\mathrm{CH}), 127.8(\mathrm{C}), 127.7(2 \times \mathrm{CH}), 127.5(\mathrm{CH}), 125.7$ $(\mathrm{CH}), 125.6(\mathrm{CH}), 66.5(\mathrm{CH}, \mathrm{C}-2), 66.4(\mathrm{CH}, \mathrm{C}-5), 60.8$ $(\mathrm{CH}, \mathrm{C}-4), 52.9\left(\mathrm{OCH}_{3}\right), 50.5(\mathrm{CH}, \mathrm{C}-3), 21.0\left(\mathrm{CH}_{3}\right), 18.1$ $\left(\mathrm{CH}_{3}\right)$. MS (ESI): m/z $499.1\left(\mathrm{MH}^{+}, 100\right), 439$ (10), 394 (3), 322 (30), 178 (77), 119 (21), 91 (5), 60 (5).

Methyl 4-(2-methylbenzoyl)-3-(2-chloroquinolin-3-yl)-5phenylpyrrolidine-2-carboxylate $(3 b)$

Yield 58\%. $\mathrm{R}_{f}\left(\mathrm{CHCl}_{3}\right): 0.34 . \mathrm{Mp} \mathrm{79-81}{ }^{\circ} \mathrm{C}$. IR (ATR) v 3347, 2926, 1735, 1676, 1567, 1488, 1454, 1331, 1202, $1133,731 \mathrm{~cm}^{-1} .{ }^{1} \mathrm{H}$ NMR $\left(300 \mathrm{MHz}, \mathrm{CDCl}_{3}\right) \delta 8.31(\mathrm{~s}, 1 \mathrm{H})$, $8.04(\mathrm{~d}, J=8.5,1 \mathrm{H}), 8.86(\mathrm{~d}, J=8.1,1 \mathrm{H}), 7.72(\mathrm{td}, J=7.7$, $J=1.1,1 \mathrm{H}), 7.59(\mathrm{t}, J=7.4,1 \mathrm{H}), 7.46(\mathrm{~d}, J=7.7,1 \mathrm{H}), 7.24(\mathrm{t}$, $J=7.6,1 \mathrm{H}), 7.17-7.11(\mathrm{~m}, 6 \mathrm{H}), 6.99(\mathrm{~d}, J=7.5,1 \mathrm{H}), 4.96(\mathrm{~d}$, $J=8.2,1 \mathrm{H}), 4.81(\mathrm{t}, J=8.1,1 \mathrm{H}), 4.70(\mathrm{t}, J=8.1,1 \mathrm{H}), 4.37(\mathrm{~d}$, $J=8.3,1 \mathrm{H}), 3.79$ (s, 3H), 2.88 (brs, $1 \mathrm{H}), 1.83(\mathrm{~s}, 3 \mathrm{H}) .{ }^{13} \mathrm{C}$ NMR (75.4 MHz, $\left.\mathrm{CDCl}_{3}\right) \delta 201.7(\mathrm{C}=\mathrm{O}), 173.2(\mathrm{C}=\mathrm{O})$, 151.3 (C), 147.1 (C), 139.9 (C), 139.0 (C), 138.4 (C), 137.8 $(\mathrm{C}), 137.5(\mathrm{CH}), 132.8(\mathrm{C}), 132.2(\mathrm{CH}), 131.9(\mathrm{CH}), 130.9$ $(\mathrm{CH}), 129.0(\mathrm{CH}), 128.7(2 \times \mathrm{XH}), 128.6(\mathrm{CH}), 128.6(\mathrm{CH})$, $128.1(\mathrm{CH}), 127.7(2 \times \mathrm{CH}), 127.7(\mathrm{CH}), 125.8(\mathrm{CH}), 66.6$ $(\mathrm{CH}, \mathrm{C}-2), 66.3(\mathrm{CH}, \mathrm{C}-5), 61.0(\mathrm{CH}, \mathrm{C}-4), 53.0\left(\mathrm{OCH}_{3}\right)$, $50.2(\mathrm{CH}, \mathrm{C}-3), 21.0\left(\mathrm{CH}_{3}\right)$. MS (ESI): m/z $485.2\left(\mathrm{MH}^{+}\right.$, 100), 425 (8), 380 (5), 308 (28), 178 (75), 146 (7), 119 (56), 91 (8), 60(7). HRMS (ESI): $\mathrm{m} / \mathrm{z}\left[\mathrm{MH}^{+}\right]$Calcd. for $\mathrm{C}_{29} \mathrm{H}_{26} \mathrm{~N}_{2} \mathrm{O}_{3}{ }^{35} \mathrm{Cl}$ : 485.16320, found 485.1628 .

Methyl 4-(2-methylbenzoyl)-3-(2-chloro-5,8-dimethoxyquinolin-3-yl)-5-phenylpyrrolidine -2-carboxylate (3c)

Yield $71 \% . \mathrm{R}_{f}\left(\mathrm{CHCl}_{3}\right): 0.34 . \mathrm{Mp} \mathrm{153-154}{ }^{\circ} \mathrm{C}$. IR (ATR) v 3339, 2953, 1733, 1674, 1617, 1592, 1481, 1330, 1263, $1014,725 \mathrm{~cm}^{-1} .{ }^{1} \mathrm{H}$ NMR $\left(300 \mathrm{MHz}, \mathrm{CDCl}_{3}\right) \delta 8.66(\mathrm{~s}, 1 \mathrm{H})$, $7.39(\mathrm{~d}, J=7.7,1 \mathrm{H}), 7.24(\mathrm{td}, J=7.4, J=1.2,1 \mathrm{H}), 7.15-7.11$ $(\mathrm{m}, 6 \mathrm{H}), 6.98(\mathrm{~m}, 2 \mathrm{H}), 6.78(\mathrm{~d}, J=8.5,1 \mathrm{H}), 4.96(\mathrm{~d}, J=8.1$, $1 \mathrm{H}), 4.73(\mathrm{t}, J=7.9,1 \mathrm{H}), 4.72(\mathrm{t}, J=8.0,1 \mathrm{H}), 4.35(\mathrm{~d}, J=8.2$, $1 \mathrm{H}), 4.03(\mathrm{~s}, 3 \mathrm{H}), 3.98(\mathrm{~s}, 3 \mathrm{H}), 3.77(\mathrm{~s}, 3 \mathrm{H}), 3.23$ (brs, $1 \mathrm{H})$, $1.80(\mathrm{~s}, 3 \mathrm{H}) .{ }^{13} \mathrm{C}$ NMR $\left(75.4 \mathrm{MHz}, \mathrm{CDCl}_{3}\right) \delta 202.14(\mathrm{C}=\mathrm{O})$, 173.2 (C=O), 151.3 (C), 148.9 (C), 148.6 (C), 139.7 (C), 139.1 (C), 139.0 (C), $138.0(\mathrm{C}), 132.7$ (C), $132.1(\mathrm{CH})$, $131.8(\mathrm{CH}), 128.9(\mathrm{CH}), 128.7(2 \times \mathrm{CH}), 128.0(\mathrm{CH}), 127.7$ $(2 \times C H), 127.4(\mathrm{CH}), 125.7(\mathrm{CH}), 121.1(\mathrm{C}), 108.6(\mathrm{CH})$, $104.9(\mathrm{CH}), 67.1$ (CH, C-2), $66.5(\mathrm{CH}, \mathrm{C}-5), 61.0(\mathrm{CH}, \mathrm{C}-4)$, $56.5\left(\mathrm{OCH}_{3}\right), 56.1\left(\mathrm{OCH}_{3}\right), 52.9\left(\mathrm{OCH}_{3}\right), 51.0(\mathrm{CH}, \mathrm{C}-3)$, $20.9\left(\mathrm{CH}_{3}\right)$. MS (ESI): m/z $545.1\left(\mathrm{MH}^{+}, 100\right), 509(10), 485$ (5), 368 (38), 332 (3), 178 (38), 119 (15), 91 (1), 60 (1). HRMS (ESI): $\mathrm{m} / \mathrm{z}\left[\mathrm{MH}^{+}\right]$Calcd. for $\mathrm{C}_{31} \mathrm{H}_{30} \mathrm{~N}_{2} \mathrm{O}_{5}{ }^{35} \mathrm{Cl}$ : 545.18432, found 545.1839.

Methyl 4-(2-methylbenzovl)-3-(2-chloro-6-methylquinolin3-yl)-5-phenylpyrrolidine-2-carboxylate (3d)

Yield 62\%. $\mathrm{R}_{f}\left(\mathrm{CHCl}_{3}\right): 0.29 . \mathrm{Mp} \mathrm{83-85}{ }^{\circ} \mathrm{C}$. IR (ATR) v 3336, 2952, 1736, 1677, 1597, 1494, 1338, 1215, 1129, 1044, $731 \mathrm{~cm}^{-1} .{ }^{1} \mathrm{H}$ NMR $\left(300 \mathrm{MHz}, \mathrm{CDCl}_{3}\right) \delta 8.21(\mathrm{~s}, 1 \mathrm{H})$, $7.93(\mathrm{~d}, J=8.6,1 \mathrm{H}), 7.62(\mathrm{~s}, 1 \mathrm{H}), 7.57$ (dd, $J=8.7, J=1.7$, $1 \mathrm{H}), 7.43(\mathrm{~d}, J=7.7,1 \mathrm{H}), 7.26(\mathrm{td}, J=7.4, J=1.2,1 \mathrm{H}), 7.17-$ $7.14(\mathrm{~m}, 6 \mathrm{H}), 6.99(\mathrm{~d}, J=7.5,1 \mathrm{H}), 4.95(\mathrm{~d}, J=8.0,1 \mathrm{H}) 4.78(\mathrm{t}$, $J=8.0,1 \mathrm{H}), 4.67(\mathrm{t}, J=8.1,1 \mathrm{H}), 4.37(\mathrm{~d}, J=7.9,1 \mathrm{H}), 3.79(\mathrm{~s}$, $3 \mathrm{H}), 2.78$ (brs, 1H), $2.55(\mathrm{~s}, 3 \mathrm{H}), 1.83(\mathrm{~s}, 3 \mathrm{H}) ;{ }^{13} \mathrm{C} \mathrm{NMR}$ $\left(75.4 \mathrm{MHz}, \mathrm{CDCl}_{3}\right) \delta 201.8(\mathrm{C}=\mathrm{O}), 173.3(\mathrm{C}=\mathrm{O}), 150.3(\mathrm{C})$, 145.7 (C), 139.8 (C), 139.0 (C), 137.8 (C), 137.8 (C), 136.9 $(\mathrm{CH}), 133.1(\mathrm{CH}), 132.6(\mathrm{C}), 132.2(\mathrm{CH}), 131.9(\mathrm{CH}), 129.0$
$(\mathrm{CH}), 128.7(2 \times \mathrm{CH}), 128.3(\mathrm{CH}), 128.1(\mathrm{CH}), 127.8(\mathrm{C})$, $127.7(2 \times C H), 126.6(\mathrm{CH}), 125.7(\mathrm{CH}), 66.4(\mathrm{CH}, \mathrm{C}-2), 66.3$ $(\mathrm{CH}, \mathrm{C}-5), 61.0(\mathrm{CH}, \mathrm{C}-4), 52.9\left(\mathrm{OCH}_{3}\right), 50.2(\mathrm{CH}, \mathrm{C}-3)$, $22.0\left(\mathrm{CH}_{3}\right), 21.0\left(\mathrm{CH}_{3}\right)$. HRMS (ESI): m/z [MH $\left.{ }^{+}\right]$Calcd. for $\mathrm{C}_{30} \mathrm{H}_{28} \mathrm{~N}_{2} \mathrm{O}_{3}{ }^{35} \mathrm{Cl}$ : 499.17885, found 499.1793.

Methyl 4-(4-methoxybenzoyl)-3-(2-chloro-8-methylquinoline-3-yl)-5-phenylpyrrolidine-2-carboxylate (3e)

Yield $60 \% . \mathrm{R}_{f}\left(\mathrm{CHCl}_{3}\right): 0.32 . \mathrm{Mp} 108{ }^{\circ} \mathrm{C}$. IR (ATR) v 3342, 2924, 1740, 1656, 1595, 1512, 1433, 1374, 1227, 1024, 749, $697 \mathrm{~cm}^{-1} .{ }^{1} \mathrm{H}$ NMR $\left(300 \mathrm{MHz}, \mathrm{CDCl}_{3}\right) \delta 8.26(\mathrm{~s}$, $1 \mathrm{H}), 7.69(\mathrm{~d}, J=8.1,1 \mathrm{H}), 7.57-7.50(\mathrm{~m}, 3 \mathrm{H}), 7.48(\mathrm{t}, J=7.7$, $1 \mathrm{H}), 7.18-7.08(\mathrm{~m}, 5 \mathrm{H}), 6.72(\mathrm{~d}, J=8.8,2 \mathrm{H}), 4.98(\mathrm{~d}, J=8.1$, $1 \mathrm{H}), 4.72(\mathrm{t}, J=7.9,1 \mathrm{H}), 4.56(\mathrm{t}, J=7.9,1 \mathrm{H}), 4.43(\mathrm{~d}, J=8.0$, $1 \mathrm{H}), 3.83$ (s, 3H), 3.78 (s, 3H), 3.22 (brs, 1H), 2.78 (s, 3H); ${ }^{13} \mathrm{C}$ NMR (75.4 MHz, $\left.\mathrm{CDCl}_{3}\right) \delta 197.6(\mathrm{C}=\mathrm{O}), 173.6(\mathrm{C}=\mathrm{O})$, $163.6(\mathrm{C}), 150.3(\mathrm{C}), 146.3(\mathrm{C}), 138.6(\mathrm{C}), 137.4(\mathrm{CH})$, $136.8(\mathrm{C}), 132.9(\mathrm{C}), 130.8(2 \mathrm{xCH}), 130.6(\mathrm{C}), 129,5(\mathrm{CH})$, $128.6(2 \times \mathrm{CH}), 128.1(\mathrm{CH}), 127.7(\mathrm{C}), 127.6(2 \times \mathrm{CH}), 127.5$ $(\mathrm{CH}), 125.7(\mathrm{CH}), 113.7(2 \times \mathrm{CH}), 66.8(\mathrm{CH}, \mathrm{C}-2), 66.0(\mathrm{CH}$, C-5), $58.8(\mathrm{CH}, \mathrm{C}-4), 55.7\left(\mathrm{OCH}_{3}\right), 53.0\left(\mathrm{OCH}_{3}\right), 50.4(\mathrm{CH}$, C-3), $18.14\left(\mathrm{CH}_{3}\right)$. MS (ESI): m/z $515.3\left(\mathrm{MH}^{+}, 85\right), 455$ (10), 410 (8), 338 (24), 178 (100), 135 (21), 118 (10), 91 (3), 60 (3). Calcd for $\mathrm{C}_{30} \mathrm{H}_{27} \mathrm{~N}_{2} \mathrm{O}_{4} \mathrm{Cl}$ : C, 69.97; H, 5.28; N. 5.44, Found C, 69.33; H, 5.36; N, 5.39.

Methyl 4-(4-methoxybenzoyl)-3-(2-chloro-6-methoxyquinolin-3-yl)-5-phenylpyrrolidine-2-carboxylate (3f)

Yield 65\%. $\mathrm{R}_{f}\left(\mathrm{CHCl}_{3}\right): 0.28 . \mathrm{Mp} 100-103{ }^{\circ} \mathrm{C}$. IR (ATR) v 3354, 2952, 1734, 1655, 1596, 1494, 1353, 1223, 1170, 907, $700 \mathrm{~cm}^{-1}$. ${ }^{1} \mathrm{H}$ NMR (300 MHz, $\left.\mathrm{CDCl}_{3}\right) \delta 8.19(\mathrm{~s}, 1 \mathrm{H})$, $7.91(\mathrm{~d}, J=9.0,1 \mathrm{H}), 7.55(\mathrm{~d}, J=8.8,2 \mathrm{H}), 7.36(\mathrm{dd}, J=9.1$, $J=2.6,1 \mathrm{H}), 7.18-7.05(\mathrm{~m}, 6 \mathrm{H}), 6.70(\mathrm{~d}, J=8.8,2 \mathrm{H}), 4.97$ (d, $J=8.1,1 \mathrm{H}), 4.68(\mathrm{t}, J=8.0,1 \mathrm{H}), 4.40(\mathrm{t}, J=8.0,1 \mathrm{H}), 4.39(\mathrm{~d}$, $J=7.9,1 \mathrm{H}), 3.93(\mathrm{~s}, 3 \mathrm{H}), 3.83(\mathrm{~s}, 3 \mathrm{H}), 3.78$ (s, 3H), 3.10 (brs, 1H). ${ }^{13} \mathrm{C}$ NMR $\left(75.4 \mathrm{MHz}, \mathrm{CDCl}_{3}\right) \delta 197.6(\mathrm{C}=\mathrm{O}), 175.6$ $(\mathrm{C}=\mathrm{O}), 163.6$ (C), 158.7 (C), 148.6 (C), 143.1 (C), 138.5 (C), $136.0(\mathrm{CH}), 133.4(\mathrm{C}), 130.8(2 \mathrm{xCH}), 130.6(\mathrm{C}), 129.9$ $(\mathrm{CH}), 128.8(\mathrm{C}), 128.6(2 \times \mathrm{xH}), 128.2(\mathrm{CH}), 127.6(2 \times \mathrm{CH})$, $123.5(\mathrm{CH}), 113.7(2 \times \mathrm{CH}), 105.3(\mathrm{CH}), 66.7(\mathrm{CH}, \mathrm{C}-2), 66.0$ $(\mathrm{CH}, \mathrm{C}-5), 58.6(\mathrm{CH}, \mathrm{C}-4), 56.0\left(\mathrm{OCH}_{3}\right), 55.7\left(\mathrm{OCH}_{3}\right), 53.0$ $\left(\mathrm{OCH}_{3}\right), 50.3(\mathrm{CH}, \mathrm{C}-3)$. MS (ESI): m/z $531.3\left(\mathrm{MH}^{+}, 73\right)$, 471 (7), 426 (7), 366 (5), 354 (28), 178 (100), 135 (24), 118 (17), 91 (8), 60 (7). HRMS (ESI): $\mathrm{m} / \mathrm{z}\left[\mathrm{MH}^{+}\right]$Calcd. for $\mathrm{C}_{30} \mathrm{H}_{28} \mathrm{~N}_{2} \mathrm{O}_{5}{ }^{35} \mathrm{Cl}$ : 531.16868, found 531.1680.

Methyl 4-(3,4-dimethoxybenzoyl)-3-(2-chloro-8-methylquinolin-3-yl)-5-phenyl pyrrolidine -2-carboxylate (3g)

Yield 53\%. $\mathrm{R}_{f}\left(\mathrm{CHCl}_{3}\right): 0.30 . \mathrm{Mp} 115-116^{\circ} \mathrm{C}$. IR (ATR) v 3350,1758, 1653,1586, 1492, 1850, $709 \mathrm{~cm}^{-1} .{ }^{1} \mathrm{H}$ NMR $\left(300 \mathrm{MHz}, \mathrm{CDCl}_{3}\right) \delta 8.29(\mathrm{~s}, 1 \mathrm{H}), 7.64-7.00(\mathrm{~m}, 10 \mathrm{H}), 6.71$ $(\mathrm{d}, J=8.0,1 \mathrm{H}), 5.00(\mathrm{~d}, J=8.0,1 \mathrm{H}), 4.78(\mathrm{t}, J=8.0,1 \mathrm{H}), 4.60$ $(\mathrm{t}, J=8.2,1 \mathrm{H}), 4.53(\mathrm{~d}, J=8.0,1 \mathrm{H}), 3.87(\mathrm{~s}, 3 \mathrm{H}), 3.85(\mathrm{~s}$, $3 \mathrm{H}), 3.76(\mathrm{~s}, 3 \mathrm{H}), 2.78(\mathrm{~s}, 3 \mathrm{H}) .{ }^{13} \mathrm{C} \mathrm{NMR}(75.4 \mathrm{MHz}$, $\left.\mathrm{CDCl}_{3}\right) \delta 197.2(\mathrm{C}=\mathrm{O}), 173.2(\mathrm{C}=\mathrm{O}), 153.0(\mathrm{C}), 150.0(\mathrm{C})$, $148.6(\mathrm{C}), 145.8(\mathrm{C}), 137.9(\mathrm{CH}), 36.9(\mathrm{C}), 136.4(\mathrm{C}), 133.4$ $(\mathrm{C}), 130.4(\mathrm{CH}), 129.6(\mathrm{C}), 128.2(2 \mathrm{xCH}), 127.8(\mathrm{CH})$, $127.3(\mathrm{C}), 127.2(2 \times \mathrm{CH}), 127.1(\mathrm{CH}), 125.3(\mathrm{CH}), 122.9$ $(\mathrm{CH}), 110.0(\mathrm{CH}), 109.5(\mathrm{CH}), 66.2(\mathrm{CH}), 65.4(\mathrm{CH}), 58.4$ $(\mathrm{CH}), 55.9\left(\mathrm{OCH}_{3}\right), 55.7\left(\mathrm{OCH}_{3}\right), 52.6\left(\mathrm{OCH}_{3}\right), 49.1(\mathrm{CH})$, $17.7\left(\mathrm{CH}_{3}\right)$. HRMS (ESI): $\mathrm{m} / \mathrm{z} \quad\left[\mathrm{MH}^{+}\right]$Calcd. for $\mathrm{C}_{31} \mathrm{H}_{30} \mathrm{~N}_{2} \mathrm{O}_{5}{ }^{35} \mathrm{Cl}: 545.18433$, found 545.1843. 
Methyl 4-(3,4,5-trimethoxybenzoyl)-3-(2-chloro-8-methylquinolin-3-yl)-5-phenyl pyrrolid-ine-2-carboxylate (3h)

Yield 54\%. $\mathrm{R}_{f}\left(\mathrm{CHCl}_{3}\right): 0.38 . \mathrm{Mp} 209-210{ }^{\circ} \mathrm{C}$. IR (ATR) v 3354, 2949, 1732, 1648, 1494, 1250, 1185, 915, $708 \mathrm{~cm}^{-1}$. ${ }^{1} \mathrm{H}$ NMR $\left(300 \mathrm{MHz}, \mathrm{CDCl}_{3}\right) \delta 8.28(1 \mathrm{H}, \mathrm{s}), 7.71(\mathrm{~d}, J=7.9$, $1 \mathrm{H}), 7.59(\mathrm{~d}, J=7.9,1 \mathrm{H}), 7.48(\mathrm{t}, J=7.9,1 \mathrm{H}), 7.16-7.10(\mathrm{~m}$, $5 \mathrm{H}), 6.77(\mathrm{~s}, 2 \mathrm{H}), 4.95(\mathrm{~d}, J=8.1,1 \mathrm{H}), 4.71(\mathrm{t}, J=8.0,1 \mathrm{H})$, 4.42-4.44 (m, 2H), $3.87(\mathrm{~s}, 3 \mathrm{H}), 3.83(\mathrm{~s}, 3 \mathrm{H}), 3.76(\mathrm{~s}, 6 \mathrm{H})$, $3.30(\mathrm{~s}, 3 \mathrm{H}), 2.78(\mathrm{~s}, 3 \mathrm{H}),{ }^{13} \mathrm{C}$ NMR $\left(75.4 \mathrm{MHz}, \mathrm{CDCl}_{3}\right) \delta$ $198.0(\mathrm{C}=\mathrm{O}), 173.4(\mathrm{C}=\mathrm{O}), 152.5(\mathrm{C}), 149.8(\mathrm{C}), 145.9(\mathrm{C})$, $142.4(\mathrm{C}), 137.9(\mathrm{C}), 136.8(\mathrm{CH}), 136.4(\mathrm{C}), 132.4(\mathrm{C})$, $132.2(\mathrm{C}), 130.5(\mathrm{CH}), 128.3(2 \times \mathrm{CH}), 128.0(\mathrm{CH}), 127.3$ $(2 \times \mathrm{CH}), 127.2(\mathrm{C}), 127.2(\mathrm{CH}), 125.3(\mathrm{CH}), 105.8(2 \times \mathrm{CH})$, $66.4(\mathrm{CH}), 65.3(\mathrm{CH}), 60.8\left(\mathrm{OCH}_{3}\right), 58.8(\mathrm{CH}), 56.2$ $\left(\mathrm{OCH}_{3}\right), 52.7\left(\mathrm{OCH}_{3}\right), 50.0(\mathrm{CH}), 17.7\left(\mathrm{CH}_{3}\right)$. HRMS (ESI): $\mathrm{m} / \mathrm{z}\left[\mathrm{MH}^{+}\right]$Calcd. for $\mathrm{C}_{32} \mathrm{H}_{32} \mathrm{~N}_{2} \mathrm{O}_{6}{ }^{35} \mathrm{Cl}: 575.19489$, found 575.1917.

Methyl 4-(methylketone)-3-(2-chloro-6-methylquinolin-3vl)-5-phenvlpyrrolidine-2-carboxylate (3i)

Yellow oil. Yield 67\%. $\mathrm{R}_{f}\left(\mathrm{CHCl}_{3}\right)$ : 0.54. IR (ATR) v $3350,2957,1732,1657,1285,1226,1085,910,695 \mathrm{~cm}^{-1} .{ }^{1} \mathrm{H}$ NMR $\left(300 \mathrm{MHz}, \mathrm{CDCl}_{3}\right) \delta 8.24(\mathrm{~s}, 1 \mathrm{H}), 7.94(\mathrm{~d}, J=8.5,1 \mathrm{H})$, $7.68(\mathrm{~s}, 1 \mathrm{H}), 7.60(\mathrm{dd}, J=8.5, J=1.6,1 \mathrm{H}), 7.37-7.25(\mathrm{~m}, 5 \mathrm{H})$, $4.87(\mathrm{~d}, J=8.0,1 \mathrm{H}), 4.53(\mathrm{t}, J=8.1,1 \mathrm{H}), 4.35(\mathrm{~d}, J=7.9,1 \mathrm{H})$, $3.82(\mathrm{~s}, 3 \mathrm{H}), 3.60(\mathrm{dd}, J=7.9, J=4.8,1 \mathrm{H}), 2.55(\mathrm{~s}, 3 \mathrm{H}), 1.50$ $(\mathrm{s}, 3 \mathrm{H}) .{ }^{13} \mathrm{C}$ NMR $\left(75.4 \mathrm{MHz}, \mathrm{CDCl}_{3}\right) \delta 208.0(\mathrm{C}=\mathrm{O}), 172.6$ $(\mathrm{C}=\mathrm{O}), 149.6(\mathrm{C}), 145.0(\mathrm{C}), 137.4(\mathrm{C}), 136.8(\mathrm{CH}), 135.9$ (C), $132.8(\mathrm{CH}), 132.2(\mathrm{C}), 128.8(2 \times \mathrm{CH}), 128.4(\mathrm{CH})$, $127.5(\mathrm{CH}), 127.3(\mathrm{C}), 126.9(2 \times \mathrm{CH}), 126.4(\mathrm{CH}), 64.8$ $(\mathrm{CH}), 64.3(\mathrm{CH}), 63.9(\mathrm{CH}), 52.7\left(\mathrm{OCH}_{3}\right), 49.0(\mathrm{CH}), 28.3$ $\left(\mathrm{OCH}_{3}\right), 21.4\left(\mathrm{CH}_{3}\right)$. HRMS (EI): $\mathrm{m} / \mathrm{z}\left[\mathrm{M}^{+}\right]$Calcd. for $\mathrm{C}_{24} \mathrm{H}_{22} \mathrm{~N}_{2} \mathrm{O}_{3}{ }^{35} \mathrm{Cl}: 421.13190$, found 421.1329 .

Methyl 4-(methylketone)-3-(2-chloroquinolin-3-yl)-5-phenylpyrrolidine-2-carboxylate (3i)

Yellow oil. Yield 59\%. $\mathrm{R}_{f}\left(\mathrm{CHCl}_{3}\right): 0.45$. IR (ATR) $v$ $3367,1739,1651,1342,1237,1110 \mathrm{~cm}^{-1} .{ }^{1} \mathrm{H}$ NMR $(300$ $\left.\mathrm{MHz}, \mathrm{CDCl}_{3}\right) \delta 8.29(\mathrm{~s}, 1 \mathrm{H}), 8.08(\mathrm{~d}, J=8.4,1 \mathrm{H}), 7,90(\mathrm{~d}$, $J=8.1,1 \mathrm{H}), 7.73(\mathrm{t}, J=7.0,1 \mathrm{H}), 7.61(\mathrm{t}, J=7.1,1 \mathrm{H}), 7.30-7.45$ $(\mathrm{m}, 5 \mathrm{H}), 4.85(\mathrm{~d}, J=8.0,1 \mathrm{H}), 4.51(\mathrm{t}, J=8.0,1 \mathrm{H}), 4.32(\mathrm{~d}$, $J=7.9,1 \mathrm{H}), 3.83(\mathrm{~s}, 3 \mathrm{H}), 3.61(\mathrm{dd}, J=8.0, J=4.8,1 \mathrm{H}) .{ }^{13} \mathrm{C}$ NMR $\left(75.4 \mathrm{MHz}, \mathrm{CDCl}_{3}\right) \delta 207.8(\mathrm{C}=\mathrm{O}), 172.8(\mathrm{C}=\mathrm{O})$, $150.6(\mathrm{C}), 146.6(\mathrm{C}), 139.2(\mathrm{C}), 137.3(\mathrm{CH}), 136.7(\mathrm{CH})$, $132.7(\mathrm{C}), 130.6(\mathrm{CH}), 128.9(2 \times \mathrm{CH}), 128.4(\mathrm{CH}), 128.1$ $(\mathrm{CH}), 127.5(\mathrm{CH}), 127.3(\mathrm{C}), 127.0(2 \times \mathrm{CH}), 65.2(\mathrm{CH}), 65.1$ $(\mathrm{CH}), 52.7\left(\mathrm{OCH}_{3}\right), 49.2(\mathrm{CH}), 31.2(\mathrm{CH}), 28.3\left(\mathrm{CH}_{3}\right)$. HRMS (EI): $\mathrm{m} / \mathrm{z} \quad\left[\mathrm{M}^{+}\right]$Calcd. for $\mathrm{C}_{23} \mathrm{H}_{21} \mathrm{~N}_{2} \mathrm{O}_{3}{ }^{35} \mathrm{Cl}$ : 408.12407, found 408.1221.

\section{ACKNOWLEDGEMENTS}

ANDRS (Agence Nationale pour le Développement de la Recherche en Santé) and MESRES (Ministère de l'Enseignement Supérieur et de la Recherche Scientifique) are gratefully acknowledged for their financial support. AB thanks l'Agence Universitaire de la Francophonie (AUF) for financial support.

\section{REFERENCES}

[1] (a) Padwa, A.; Brodney, M. A.; Liu, B.; Satake, K.; Wu, T. J. Org. Chem. 1999, 64, 3595. (b) Egan, T. J. J. Inorg. Biochem. 2006, 100, 916. (c) Sahu, N. P.; Pal, C.; Mandal, N. B.; Banerjee, S.;
Raha, M.; Kundu, A. P.; Basu, A.; Ghosh, M.; Roy, K.; Bandyopadhyay, S. Bioorg. Med. Chem. 2002, 10, 1687.

(a) Robert, A.; Meunier, B. Chem. Soc. Rev. 1998, 27, 273. (b) Dow, G. S.; Heady, T. N.; Bhattacharjee, A. K.; Caridha, D.; Gerena, L.; Gettayacamin, M.; Lanteri, C. A.; Obaldia, N.; Roncal, N.; Shearer, T.; Smith, P. L.; Tungtaeng, A.; Wolf, L.; Cabezas, M.; Yourick, D.; Smith, K. S. Antimicrob. Chemother. 2006, 50, 4132. (c) Tjitra, E.; Baker, J.; Suprianto, S.; Cheng, Q.; Anstey N. M. Antimicrob. Agents Chemother. 2002, 46, 3947. (d) Sowunmi, A.; Sowunmi, C. O.; Adedeji, A. A.; Oduold, A. M. Clin. Drug Invest. 2001, 21, 33 .

[3] (a) Tsuge, O.; Kanemasa, S. Advances in Heterocyclic Chemistry, Katritzky, A. R., Ed.; Academic: San Diego, 1989; Vol. 45, p 231. (b) Gothelf, K. V. In "Cycloaddition Reactions in Organic Synthesis" Kobayashi, S.; Jørgensen, K. A.; Eds. Wiley-VCH, Weinheim, 2002, Chapter 6, pp. 211-247.

[4] For some recent references on this subject see: (a) Fraley, M. E.; Hartman, G. D.; Hungate, R. W. WO 0162252, August 30, 2001. (b) Arrington, K. L.; Bilodeau, M. T.; Fraley, M.; Hartman, G. D.; Hoffman, W. F.; Hungate, R.; Kim, Y. WO 0129025, April 26, 2001. (c) Fraley, M. E.; Hambaugh, S. R.; Hungate, R . W. WO 0128993, April 26, 2001. (d) Witherup, K.; Ranson, R. W.; Graham, A. C.; Barnard, A. M.; Salvatore, M. J.; Limma, W. C.; Anderson, P. S.; Pitzenberger, S. M.; Varga, S. L. J. Am. Chem. Soc. 1995, 117, 6682. (e) Kravchenko, D. V.; Kysil, V. M.; Tkachenko, S. E.; Maliarchouk, S.; Okun Ilya, M. 1.; Ivachtchenko, A. V. Eur. J. Med. Chem. 2005, 40, 1377. (f) Alcaide, B and Almendros, P. Angew. Chem. Int. Ed. 2003, 42, 858.

[5] (a) Bouraiou, A.; Menasra, H.; Debache, A.; Rhouati, S.; Belfaitah, A. J. Soc. Alger. Chim. 2006, 16, 171. (b) Kedjadja, A.; Moussaoui, F.; Debache A.; Rhouati S.; Belfaitah A. J. Soc. Alger. Chim. 2004, $14,225$.

[6] Bouraiou, A.; Debache, A.; Rhouati, S.; Carboni, B.; Belfaitah, A. J. Heterocycl. Chem. 2008, 45, 329.

[7] For a similar approach using azomethine ylides generated from Nalkylamino acids and formaldehyde, see: Menasra, H.; Kedjadja, A.; Rhouati, S.; Carboni, B.; Belfaitah, A. Synth. Commun. 2005, $35,2779$.

[8] (a) Rezig, R.; Chebah, M.; Rhouati, S.; Ducki, S.; Lawrence, N. J. Soc. Alger. Chim. 2000, 10, 111. (b) Moussaoui, F.; Belfaitah, A.; Debache, A.; Rhouati, S. J. Soc. Alger. Chim. 2002, 12, 71.

[9] (a) Tsuge, O.; Kanemasa, S.; Yoshioka, M. J. Org. Chem. 1988, 53, 1384. (b) Butler, R. N.; Farrell, D. M. J. Chem. Res. (S). 1998, 2, 82. (c) Annunziata, R.; Cinquina, M.; Cozzi, M.; Raimondi, L.; Pilati, T. Tetrahedron Asymmetry 1991, 2, 1329, and references cited therein.

[10] (a) Lown, J. W. In "1,3- Dipolar Cycloaddition Chemistry", Padwa, A.; Ed. Wiley, New York, 1984, Vol. 1, p. 653. (b) Coldham, L.; Collis, A. J.; Mould, R. J.; Robinson, D. E. Synthesis 1995, 9, 1147. (c) Witttland, C.; Arend, M.; Rish, N. Synthesis 1996, 3, 367. (d) Galley, G.; Liebsher, J.; Pätzel, M. J. Org. Chem. 1995, 60, 5005. (e) Nyerges, M.; Rudas, M.; Tóth, G.; Herényi, B. Bitter, I.; Tőke, L. Tetrahedron 1995, 51, 13321.

[11] (a) Grigg, R.; Montgomery, J.; Somasunderam, A. Tetrahedron 1992, 48, 10431. (b) Pak, C. S.; Nyerges, M. Bull. Korean Chem. Soc. 1999, 20, 633.

[12] (a) Pak, C. S.; Nyerges, M. Synlett 1999, 8, 1271. (b) Nyerges, M.; Rudas, M.; Bitter, I.; Tőke, L. Tetrahedron 1997, 53, 3269. (c) Fejes, I.; Töke, L.; Blaskó, G.; Nyerges, M.; Pak, C. S. Tetrahedron 2000, 56, 8545.

[13] Belfaitah, A.; Isly, M.; Carboni, B. Tetrahedron Lett. 2004, 45, 1969.

[14] The single-crystal growth was carried out in $\mathrm{CHCl}_{3}$ at room temperature. X-ray diffraction data have been collected with an EnrafNonius KAPPA CCD at $293 \mathrm{~K}$ using Mo K $\alpha$ radiation $(\lambda=0.71073$ $\AA$ ). The crystal $3 \mathrm{e}$ belong to the triclinic space group $P_{-1}$, with unitcell parameters $a=12.4982(2) \AA, \quad b=13.6473(4) \AA, \quad c$ $18.3062(2) \AA, \alpha=110.709(3)^{\circ}, \quad \beta=105.647(4)^{\circ}$ and $\gamma=$ $89.9690(10)^{\circ}$. The asymmetric unit contains two molecules. Bond angles, bond distances and other crystallographic data (excluding structure factors) for the structures reported in this paper have been deposited with the Cambridge Crystallographic Data Centre as supplementary Publication No. CCDC-662754 for compound 3e. Copies of the data can be obtained free of charge on application to the CCDC, 12 Union Road, Cambridge, CB2 1EZ, UK. 
[15] (a) Meth-Cohn, O.; Rhouati, S.; Tarnowski, B.; Robinson, A. J. Chem. Soc. Perkin Trans. 1981, 1, 1537. (b) Tóth, J.; Blaskó, G.; Dancsó, A.; Töke, L.; Nyerges, M. Synth. Commun. 2006, 3581.
[16] Stork, G.; Leong, A. W.; Touzin, A. M. J. Org. Chem. 1976, 41, 3491 .

(C) Bouraiou et al.; Licensee Bentham Open.

This is an open access article licensed under the terms of the Creative Commons Attribution Non-Commercial License (http://creativecommons.org/licenses/by-nc/3.0/) which permits unrestricted, non-commercial use, distribution and reproduction in any medium, provided the work is properly cited. 